\title{
Parallel Magnetic Resonance Imaging Using Compressed Sensing
}

\author{
Ali Bilgin ${ }^{\text {a,b* }}$, Yookyung Kim ${ }^{\mathrm{a}}$, Hariharan G. Lalgudi ${ }^{\mathrm{a}}$, Theodore P. Trouard ${ }^{\mathrm{c}}$, Maria I. Altbach ${ }^{\mathrm{b}}$ \\ ${ }^{a}$ Department of Electrical and Computer Engineering, University of Arizona, Tucson, AZ 85721 \\ ${ }^{\mathrm{b}}$ Department of Radiology, University of Arizona, Tucson, AZ 85724 \\ ${ }^{c}$ Department of Biomedical Engineering, University of Arizona, Tucson, AZ 85721
}

\begin{abstract}
Although magnetic resonance imaging (MRI) is routinely used in clinical practice, long acquisition times limit its practical utility in many applications. To increase the data acquisition speed of MRI, parallel MRI (pMRI) techniques have recently been proposed. These techniques utilize multi-channel receiver arrays and are based on simultaneous acquisition of data from multiple receiver coils. Recently, a novel framework called Compressed Sensing (CS) was introduced. Since this new framework illustrates how signals can be reconstructed from much fewer samples than suggested by the Nyquist theory, it has the potential to significantly accelerate data acquisition in MRI. This paper illustrates that CS and pMRI techniques can be combined and such joint processing yields results that are superior to those obtained from independent utilization of each technique.
\end{abstract}

Keywords: magnetic resonance imaging, parallel imaging, compressed sensing, compressive sampling

\section{INTRODUCTION}

MRI is an imaging modality widely used for clinical diagnosis. While MRI has many desirable properties such as lack of radiation, non-invasiveness and 3D imaging capability, long data acquisition times reduce the practical utility of this medical imaging modality in some applications. Thus, reduction of imaging time has been an important area of research in MRI. Advances in gradient technology have been a key factor in development of faster imaging techniques over the past few decades. In addition, recent introduction of pMRI methods have provided substantial increase in imaging speed. pMRI techniques exploit the use of multichannel receiver arrays and acquire the magnetic resonance (MR) signal simultaneously from several receiver coils that have varying spatial sensitivity. Compared to single coil acquisition schemes, the speed of image acquisition with pMRI is increased by only acquiring a fraction of the data necessary to reconstruct a full FOV image. This is possible because the spatial sensitivity information of the receiver coils is also used to reconstruct the image from this reduced dataset. While the most commonly known pMRI methods include SMASH [1], SENSE [2], and GRAPPA [3], there have been many other techniques proposed over the last decade. A good review of pMRI techniques can be found in [4][5]. Despite their recent introduction, the pMRI methods were quickly embraced by the MR community. These methods were rapidly and widely commercialized. They are currently available on all commercial scanners and are used routinely in clinic practice.

Recently, Candès et al. [6] and Donoho [7] introduced a mathematical framework for reconstructing sparse or compressible signals from a small number of incoherent linear measurements. This new framework is referred to as Compressive Sampling or Compressed Sensing. The CS theory illustrates that signals that have a sparse representation in a known basis can be reconstructed from a much smaller number of measurements compared to the number of measurements suggested by the Nyquist theorem. Instead of describing sufficient

\footnotetext{
*Corresponding Author: Ali Bilgin (bilgin@ieee.org).
} 
sampling based on the band-limitedness of signals as in Nyquist theory, the CS theory describes sufficient sampling based on the compressibility of signals. MRI represents a good application for CS theory since imaging is performed by making linear measurements (in Fourier space) of the object and MR images are highly compressible. Recent results indicate that CS theory can be applied to MRI and yield significantly accelerated data acquisition [8][9][10][11][12][13][14][15][16].

In this work, it is illustrated that pMRI and CS can be combined. This paper is organized as follows: In Section 2, a brief overview of pMRI and CS methods in MRI is provided. In Section 3, the proposed method that combines pMRI and CS is introduced. Experimental results and conclusions are presented in Section 4, and Section 5, respectively.

\section{THEORY}

\subsection{Parallel Imaging in MRI: SENSE and CG-SENSE}

In this section, two pMRI methods referred to as SENSE [2] and Conjugate Gradient SENSE (CG-SENSE) [17] are discussed. A conventional, fully sampled data acquisition is illustrated in Figure 1(a). Here, sufficient samples are acquired in Fourier space so that the image can be reconstructed by simply taking the inverse Fourier transform of the acquired data. The pMRI data acquisition corresponding to a reduction factor $R=2$ is illustrated in Figure 1(b). Since every other Fourier line along the y direction is skipped during data acquisition, the inverse Fourier transform of the acquired data results in a reduced FOV image with aliasing in this case. The SENSE technique is an unaliasing method applied to the reduced FOV images. SENSE is based on the observation that for a given undersampled Fourier trajectory (such as the one in Figure 1b), it is possible to determine which pixels in the full FOV image have been superimposed to form a given pixel in the reduced FOV image. SENSE uses the knowledge of the aliasing pattern together with the sensitivity profile of each coil to obtain the full FOV image. Let $f(x, y)$ denote the signal of interest at a certain location $(x, y)$, and let $S_{k}(x, y)$ denote the sensitivity profile of the k-th receiver coil at the same location. The reduced FOV image for the $\mathrm{k}$-th receiver coil can then be expressed as

$$
I_{k}(x, y)=S_{k}\left(x, y_{1}\right) f\left(x, y_{1}\right)+S_{k}\left(x, y_{2}\right) f\left(x, y_{2}\right)+\ldots+S_{k}\left(x, y_{R}\right) f\left(x, y_{R}\right)
$$

where $y_{i}, i=1, \ldots, R$ denote the y-coordinates of the pixels in the full FOV image that were superimposed to form the pixel at location $(x, y)$ in the reduced FOV image. The set of linear equations for all $n_{c}$ coils can be expressed in matrix notation

$$
\mathbf{I}_{x y}=\mathbf{S}_{x y} \mathbf{f}_{x y}
$$

where $\mathbf{I}_{x y}$ is an $n_{c}$-dimensional vector consisting of the values of the reduced FOV image at the chosen pixel for each coil, $\mathbf{S}_{x y}$ is an $n_{c} \times R$ matrix whose entries correspond to the coil sensitivities at the $\mathrm{R}$ superimposed locations, and $\mathbf{f}_{x y}$ is an $R$-dimensional vector of the desired pixel values in the full FOV image. If the values of the sensitivity matrix $\mathbf{S}_{x y}$ are known or can be estimated, the pixel values of the full FOV image can be recovered using

$$
\mathbf{f}_{x y}=\mathbf{S}_{x y}^{\mathrm{H}} \mathbf{S}_{x y}{ }^{-1} \mathbf{S}_{x y}^{\mathrm{H}} \mathbf{I}_{x y}
$$

By solving Equation (3) for each of the pixels in the reduced FOV image, all of the pixel values in the full FOV image can be recovered. 

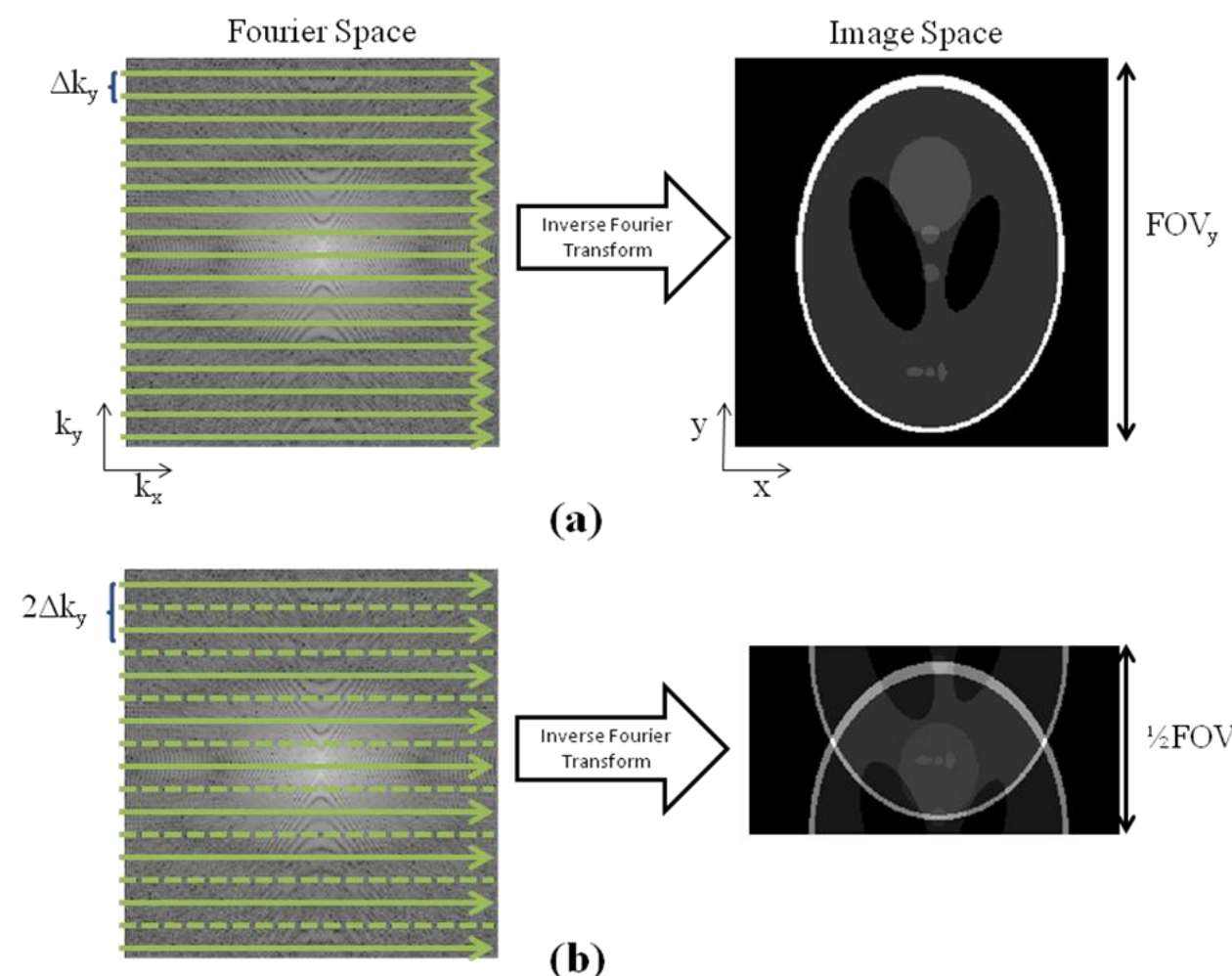

(a)

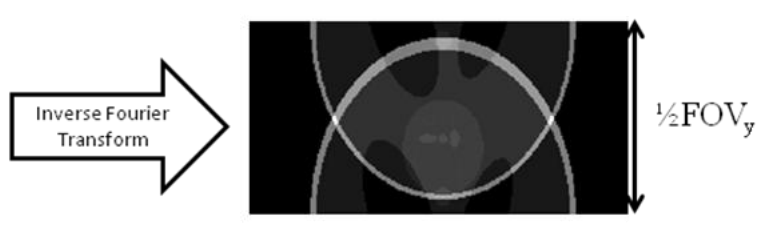

(b)

Figure 1: (a) Fully sampled data acquisition and the resulting full FOV image. (b) pMRI acquisition corresponding to a reduction factor $\mathrm{R}=2$ and the resulting reduced FOV image with aliasing artifacts. The solid lines indicate acquire Fourier lines and the dashed lines indicate the unacquired lines.

While the SENSE method works well for the Cartesian sampling case described above, its direct application to more general sampling patterns such as spiral or radial trajectories is difficult. This is because the aliasing that results from undersampling arbitrary Fourier trajectories is significantly more complex than the uncomplicated Cartesian case and simple unfolding of these superimposition patterns in the image domain is difficult. To address these problems, an iterative method called conjugate gradient SENSE (CG-SENSE) was proposed [17]. In CG-SENSE, the reconstruction problem is formulated using the equation

$$
\mathbf{E}^{\mathrm{H}} \mathbf{E} \mathbf{f}=\mathbf{E}^{\mathrm{H}} \mathbf{g}
$$

where $\mathbf{f}$ is an $N^{2}$-dimensional vector of the desired pixel values in the $N \times N$ full FOV image, and $\mathbf{g}$ is an $n_{c} n_{K}$-dimensional vector containing the $n_{K}$ measurements made by each of the $n_{c}$ receiver coils in Fourier space. $\mathbf{E}$ is an $n_{c} n_{K} \times N^{2}$ encoding matrix whose entries are given by

$$
E_{l k, j}=e^{i \mathbf{k}_{k} \mathbf{r}_{j}} s_{l}\left(\mathbf{r}_{j}\right)
$$

where $\mathbf{r}_{j}$ denotes the position of the $\mathrm{j}$-th pixel, $\mathbf{k}_{k}$ is the k-th sampling position in Fourier space, and $s_{l}\left(\mathbf{r}_{j}\right)$ is the sensitivity of the 1-th coil at pixel location j. In CG-SENSE, Equation (4) is solved iteratively using the conjugate gradient method [18]. To improve the conditioning of the problem in Equation (4), regularization techniques have been proposed [19][20][21]. The Tikhonov regularized problem can be stated as

$$
\min _{\mathbf{f}}\left(\|\mathbf{g}-\mathbf{E} \mathbf{f}\|_{2}+\lambda^{2}\|\mathbf{f}\|_{2}\right)
$$


In Equation (6), the regularization parameter $\lambda$ allows for adjusting the relative importance of the two functionals and, thus, is used to trade off between agreement with measured data and noise amplification. One method of determining the optimal parameter $\lambda$ is the L-curve algorithm [22].

It is important to note that both SENSE and CG-SENSE require explicit knowledge of the sensitivity profiles of each coil. Different methods have been proposed in the literature for estimating the sensitivity profiles. In [2], Pruessmann et. al. propose an additional calibration scan for coil sensitivity estimation. During this scan, data is acquired using both the receiver coil array and another coil with homogeneous sensitivity such as the body coil. The images obtained from each coil are then divided by the image from the coil with the homogeneous sensitivity to obtain the coil sensitivities. One of the disadvantages of this approach is its sensitivity to motion. If the patient or the coil array moves between the calibration scan and the accelerated imaging, the sensitivity estimation can be inaccurate. An alternative approach was proposed in [23] where the fully sampled central region of a variable-density Fourier acquisition was used to extract the sensitivity information.

\subsection{Compressed Sensing}

The recently introduced CS theory illustrates how a signal that has a sparse representation can be recovered from a small number of random linear measurements [6][7]. Let $\mathbf{f} \in \mathbb{C}^{L}$ denote the L-dimensional discrete complex signal of interest and let $\mathbf{g} \in \mathbb{C}^{K}$ denote the $\mathrm{K}$ linear measurements made on this signal. If $\mathbf{M}$ denotes the $K \times L$ measurement matrix, the measurement system can be represented as

$$
\mathbf{g}=\mathbf{M f}
$$

Since $\mathbf{f}$ is known to have a sparse representation, let $\boldsymbol{\Psi}$ denote the orthogonal transform matrix that represents $\mathbf{f}$ in the sparsity basis. Thus, $\boldsymbol{\Psi} \mathbf{f}$ is sparse and the support of $\mathbf{f}$ in the sparsity basis is denoted as $S,|S|<<L$. The primary result in CS theory states that when the entries of the measurement matrix $\mathbf{M}$ are selected at random, the signal $\mathbf{f}$ can be recovered with very high probability from $K \geq O(|S| \log (L))$ measurements by solving the convex optimization problem:

$$
\min _{\mathbf{f}}\left(\|\mathbf{\Psi f}\|_{1}\right) \text { such that }\|\mathbf{g}-\mathbf{M f}\|_{2} \leq \varepsilon
$$

where the parameter $\varepsilon$ is used to account for noise in the system. Equation (8) can be converted to an unconstrained optimization problem

$$
\min _{\mathbf{f}}\left(\|\mathbf{g}-\mathbf{M f}\|_{2}+\lambda^{2}\|\mathbf{\Psi} \mathbf{f}\|_{1}\right)
$$

and solved iteratively using the conjugate gradient method [15]. In Equation (9), the regularization parameter $\lambda$ allows for trading off between data consistency and sparsity.

More recent results in CS theory indicate that completely random sampling may not be necessary [24]. Instead, it is required that the undersampled measurements lead to incoherent aliasing artifacts in the sparsity space. This is important, since completely random Fourier sampling is generally impractical in MRI. Recent results indicate that CS theory can be applied to MRI using undersampled radial [11][12][13][14][16], spiral [8], and 3DFT trajectories [10]. When used in MRI, the measurement matrix $\mathbf{M}$ in Equation (9) is an $n_{K} \times N^{2}$ (undersampled) Fourier matrix whose entries are given by

$$
M_{k, j}=e^{i \mathbf{k}_{k} \mathbf{r}_{j}}
$$

In addition, it was shown that the discrete wavelet transform and finite differences (i.e. Total Variation (TV)) are good choices for the sparsity transform $\boldsymbol{\Psi}$ in MRI applications [15]. 
Incoherent aliasing is important in CS, since CS theory states that the more incoherent a measurement/sparsity system is the fewer measurements are needed for perfect recovery [25]. One method of measuring incoherence is to use transform point spread function (TPSF) analysis [15]. TPSF is defined as

$$
\operatorname{TPSF}_{C S}(m, n)=\mathbf{e}_{n}^{H} \boldsymbol{\Psi} \mathbf{M}^{\mathrm{H}} \mathbf{M} \Psi^{\mathbf{H}} \mathbf{e}_{m}
$$

where $\mathbf{e}_{m}$ denotes the $\mathrm{m}$-th vector of the natural basis. Intuitively, $\operatorname{TPSF}_{C S}(m, n)$ is the interference of a transform coefficient of unit length at location $m$ with the transform coefficient at location $n$ due to the undersampling in the measurement system. In CS, it is desired that $\operatorname{TPSF}_{C S}(m, n), m \neq n$ is both small in magnitude and has noise-like statistics. The maximum of the sidelobe-to-peak ratio (SPR) of the TPSF

$$
\max _{C S-S P R}=\max _{m \neq n}\left|\frac{\operatorname{TPSF}_{C S}(m, n)}{T P S F_{C S}(m, m)}\right|
$$

can be used as a measure of the worst case interference due to the undersampling in the measurement system. The standard deviation of the TPSF, $\sigma_{C S-T P S F}$, can be used to measure incoherence as well.

\section{PARALLEL COMPRESSED SENSING}

As discussed in the previous section, both parallel imaging and CS are techniques used to accelerate the imaging process in MRI. While parallel imaging exploits the knowledge of the coil sensitivities, CS exploits the inherent sparsity of MR images to enable reconstruction of undersampled data. The goal of this work is to combine these techniques by jointly exploiting the knowledge of the coil sensitivities and the sparsity of MR images to achieve improved performance. The combined parallel imaging and CS (PICS) reconstruction can be formulated as an unconstrained optimization problem:

$$
\min _{\mathbf{f}}\left(\|\mathbf{g}-\mathbf{E f}\|_{2}+\lambda^{2}\|\mathbf{\Psi f}\|_{1}\right)
$$

By comparing Equation (9) to Equation (13), it can be seen that the difference between CS and PICS is in the measurement matrix. In the CS case, the entries of the measurement matrix are given in Equation (10). The entries of the measurement matrix for the PICS case are given in Equation (5). Due to the joint processing of all the measurements from the coil array, the number of measurements in the PICS case is increased by the number of coils compared to the CS case. In addition, the measurements in the PICS case are modulated by the sensitivity profiles of the coils. While increased number of measurements generally allows recovery of more transform coefficients in the sparsity domain and result in increased performance, the increase in the number of recovered transform coefficients is also dependent on the sensitivity profiles of the receiver coil array. The incoherency of the aliasing artifacts in the PICS case can also be analyzed using TPSF analysis. In this case, the TPSF PICS $_{\text {is defined as }}$

$$
\operatorname{TPSF}_{\text {PICS }}(m, n)=\mathbf{e}_{n}^{H} \boldsymbol{\Psi} \mathbf{E}^{\mathrm{H}} \mathbf{E} \boldsymbol{\Psi}^{\mathbf{H}} \mathbf{e}_{m}
$$

The quantities $\max _{P I C S-S P R}$ and $\sigma_{\text {PICS-TPSF }}$ can be calculated by replacing TPSF $F_{C S}$ with TPSF $F_{\text {PICS }}$ in the calculation of $\max _{C S-S P R}$ and $\sigma_{C S-T P S F}$, respectively.

\section{RESULTS}

In this section, the performance of PICS is investigated and compared to that of CS, SENSE, and CG-SENSE. In our analysis, we use the radial Fourier trajectory because of its desirable properties such as motion insensitivity [26] and variable sampling density. However, the framework presented here can be used for other trajectories as well. In our results, we use a $256 \times 256$ image matrix, and 256 sampling points along 
each radial line in Fourier domain. The number of radial lines is adjusted to achieve the desired amount of undersampling. A brain dataset obtained using an 8-channel head coil was used to create the coil sensitivities to be used during the computer simulations. The fully sampled central region of the Fourier data for each coil was reconstructed and combined using sum-of-squares. These individual low-resolution coil images were then divided by the sum-of-squares image to estimate the coil sensitivities. These sensitivity profiles are illustrated in Figure 2.

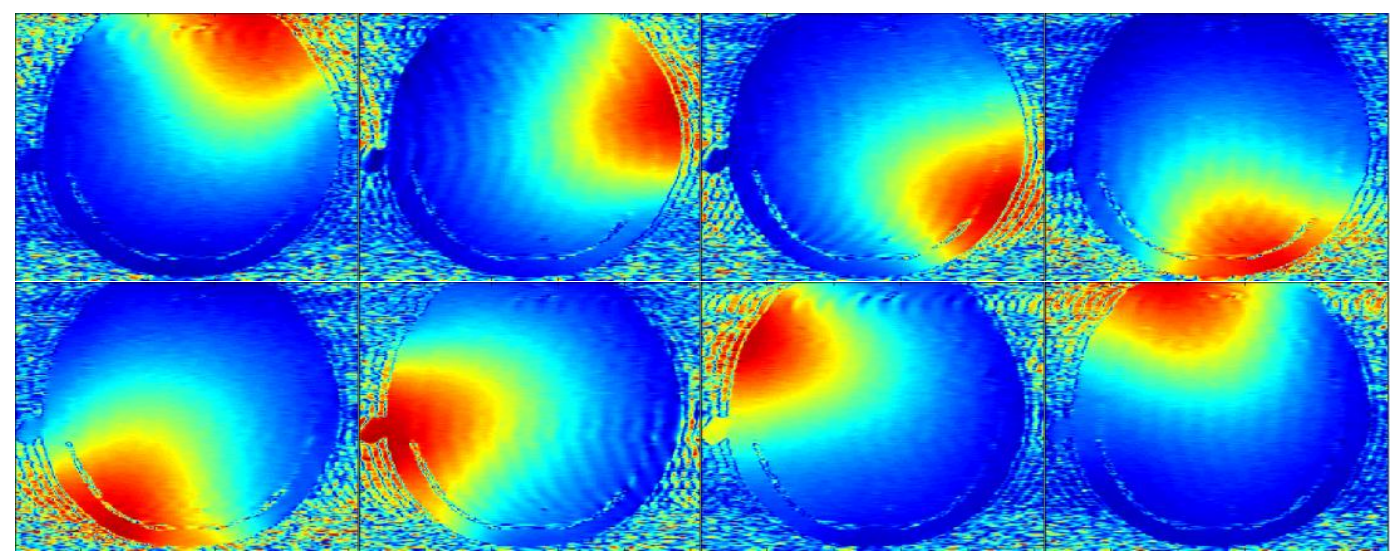

Figure 2: Coil sensitivities used in computer simulations.

\subsection{TPSF Analysis}

We first compare the incoherency properties of CS and PICS. The results in this section are obtained using a radial trajectory with 32 views. The sparsifying transform used in this section is the 3-level Daubechies-6 wavelet transform. In Figure 2, $T P S F_{C S}(m, n)$ and $\operatorname{TPSF}_{P I C S}(m, n)$ are visualized for a particular value of $m$ that corresponds to a location in the $\mathrm{HH}_{1}$ subband. We can see that the interference due to undersampling is spread within the wavelet subband, across other subbands at the same resolution level, and across other resolution levels in both cases. However, the interference is smaller for the PICS case compared to CS.
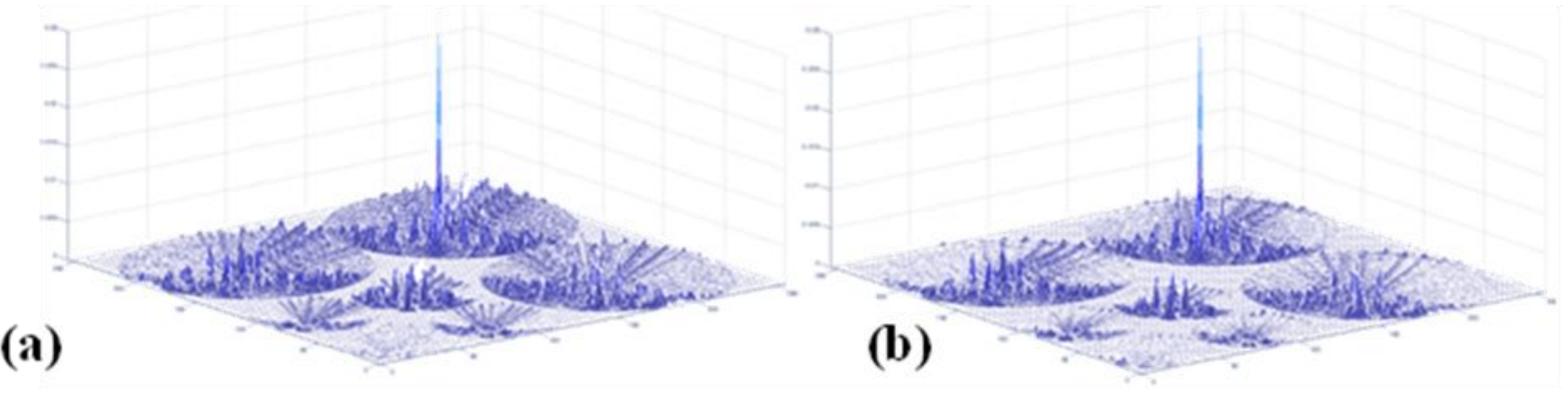

Figure 3: (a) $T P S F_{C S}(m, n)$ (b) $T P S F_{P I C S}(m, n)$ for one value of $m$ that corresponds to a location in the $\mathrm{HH}_{1}$ subband.

Our analysis indicates that the value of $\max _{P I C S-S P R}$ is smaller than $\max _{C S-S P R}$ by about \%8. A closer, subband-by-subband analysis indicates that this decrease ranges between \%0-\%11 between subbands. These results indicate that the worst-case performance improvement of PICS over CS is expected to be modest in this case. On the other hand, $\sigma_{P I C S-T P S F}$ is smaller than $\sigma_{C S-T P S F}$ by a larger margin. In Table 1, we present the percent decrease in the standard deviation of SPR between PICS and CS defined as 


$$
\% \Delta=100 \times\left(\sigma_{C S-T P S F}-\sigma_{P I C S-T P S F}\right) / \sigma_{C S-T P S F}
$$

\begin{tabular}{|c|c|c|c|c|c|c|c|c|c|c|}
\hline & $\mathrm{LL}_{3}$ & $\mathrm{HL}_{3}$ & $\mathrm{LH}_{3}$ & $\mathrm{HH}_{3}$ & $\mathrm{HL}_{2}$ & $\mathrm{LH}_{2}$ & $\mathrm{HH}_{2}$ & $\mathrm{HL}_{1}$ & $\mathrm{LH}_{1}$ & $\mathrm{HH}_{1}$ \\
\hline$\% \Delta$ & 22.95 & 39.93 & 29.85 & 19.54 & 24.01 & 29.43 & 26.89 & 26.85 & 31.34 & 28.76 \\
\hline
\end{tabular}

Table 1: Percent decrease in the standard deviation value of SPR for PICS compared to CS.

\subsection{Phantom Results}

A computer generated Shepp-Logan phantom was used to compare different methods. The phantom image was modulated using the eight sensitivity profiles shown in Figure 2. For each simulated coil, samples were taken in Fourier space along a radial trajectory. White Gaussian noise was then added independently to both real and imaginary parts of the Fourier data. Noise was uncorrelated between different coils. Images were then reconstructed using different methods. In Figure 4, images reconstructed using 16 radial views $(\sim 24 \mathrm{X}$ undersampling) are presented. The image in Figure 4(a) was obtained by reconstructing each coil image using Non-Uniform Fourier Transform (NUFFT) [27] and combining the coil images using sum-of-squares. The images in Figures 4(b) and 4(c) were obtained using CG-SENSE and CG-SENSE with Tikhonov regularization, respectively. The image obtained using CS reconstruction followed by sum-of-squares combination is shown in Figure 4(d), and the PICS image is shown in Figure 4(e). For CS and PICS, finite differences was used as the sparsifying transform. Similarly, images reconstructed using 32 radial views $(\sim 12 \mathrm{X}$ undersampling) are presented in Figure 5.

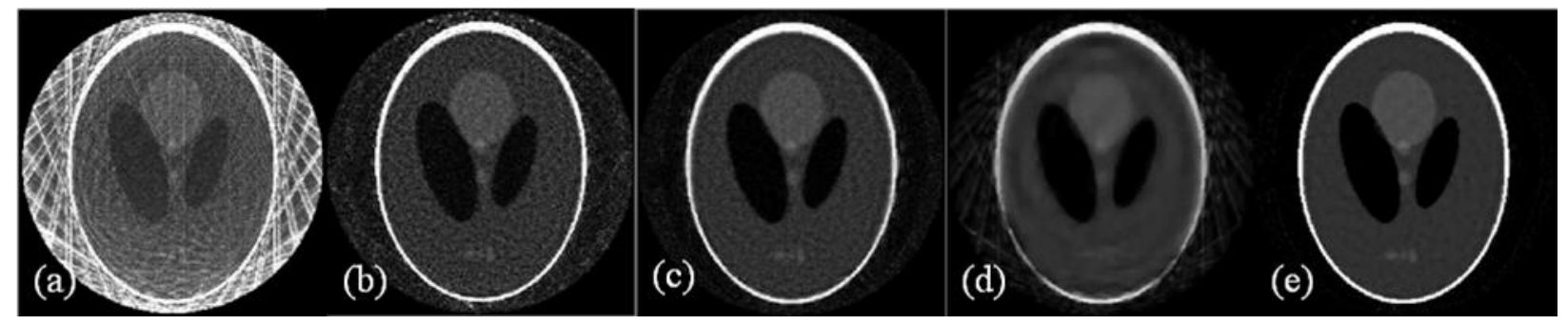

Figure 4: Phantom images reconstructed from 16 radial views using (a) NUFFT+SOS (b) CG-SENSE (c) CG-SENSE with Tikhonov regularization (d) CS + SOS (e) PICS.

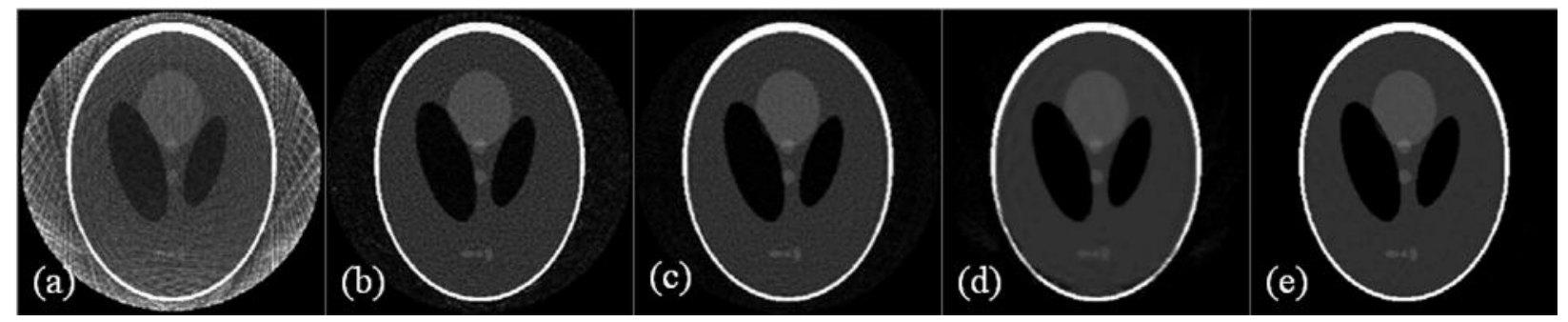

Figure 5: Phantom images reconstructed from 32 radial views using (a) NUFFT+SOS (b) CG-SENSE (c) CG-SENSE with Tikhonov regularization (d) CS + SOS (e) PICS. 


\subsection{In vivo Results}

A radial Fast Spin Echo data set acquired with a 1.5T clinical scanner (GE Healthcare, Waukesha, WI) and an 8 -channel head coil array was used to obtain in vivo results. Acquisition parameters were TR $=4.5 \mathrm{sec}$, $\mathrm{FOV}=28 \mathrm{~cm}, \mathrm{ETL}=4$. The full radial dataset consisted of 256 equally spaced radial lines with 256 sample points each. A reference image was obtained by using the full dataset and reconstructing each coil image using NUFFT and combining the coil images using sum-of-squares. This reference image is presented in Figure 6(a). The dataset was then subsampled and only 32 equally spaced radial lines were kept $(\sim 12 \mathrm{X}$ undersampling). Images were reconstructed from the subsampled dataset using different methods. The results are presented in Figures 6(b)-6(f) for NUFFT+ sum-of-squares, CG-SENSE, CG-SENSE with Tikhonov regularization, CS, and PICS, respectively. For CS and PICS, 3-level Daubechies-6 wavelet transform and finite differences were used as the sparsifying transforms. Similarly, images reconstructed using 64 radial views ( $\sim \mathrm{X}$ undersampling) are presented in Figure 7.

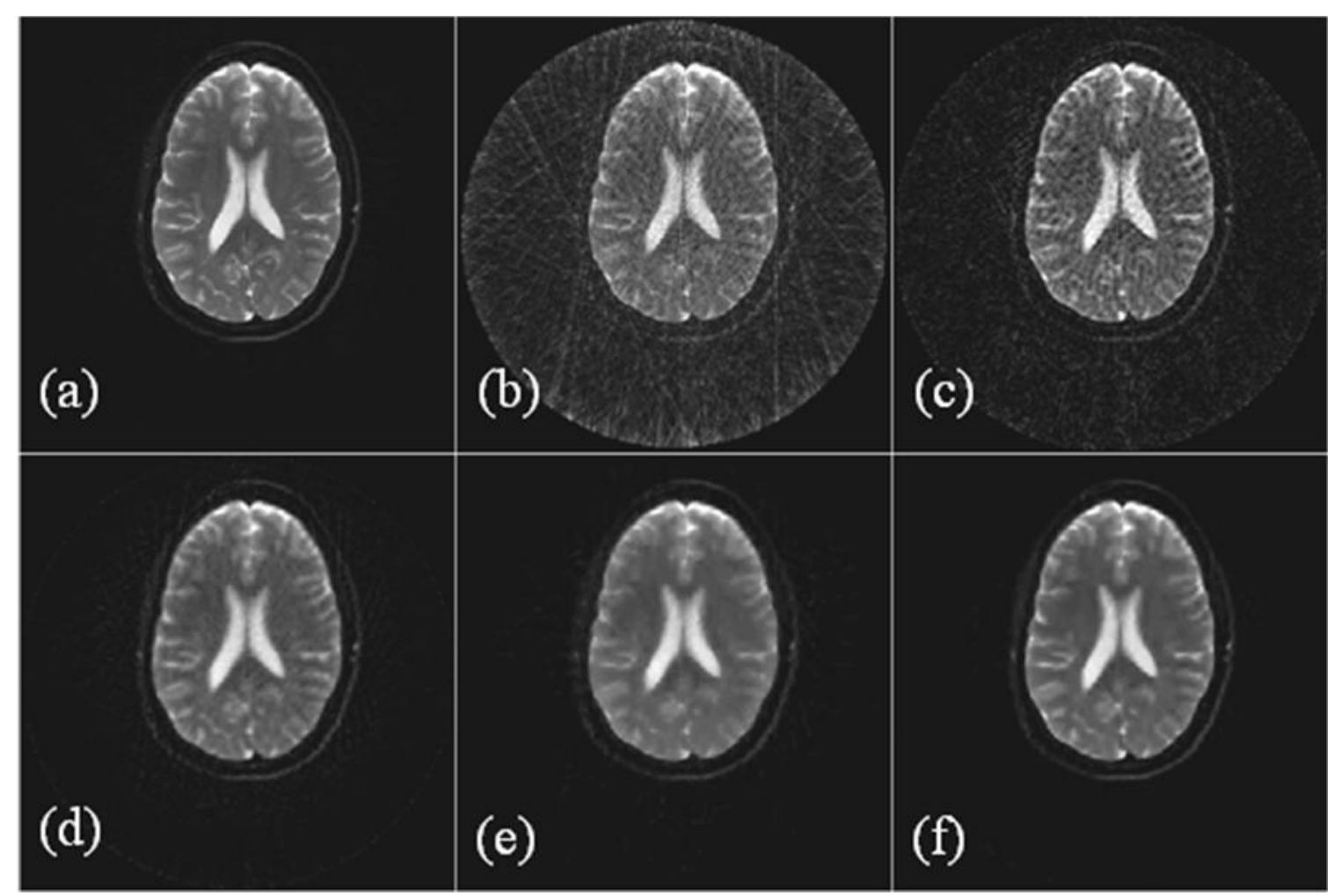

Figure 6: In vivo images reconstructed using different methods. (a) NUFFT+SOS from 256 radial views (b) NUFFT+SOS from 32 radial views (c) CG-SENSE (d) CG-SENSE with Tikhonov regularization (e) CS + SOS (f) PICS. (c)-(e) uses 32 radial views. 


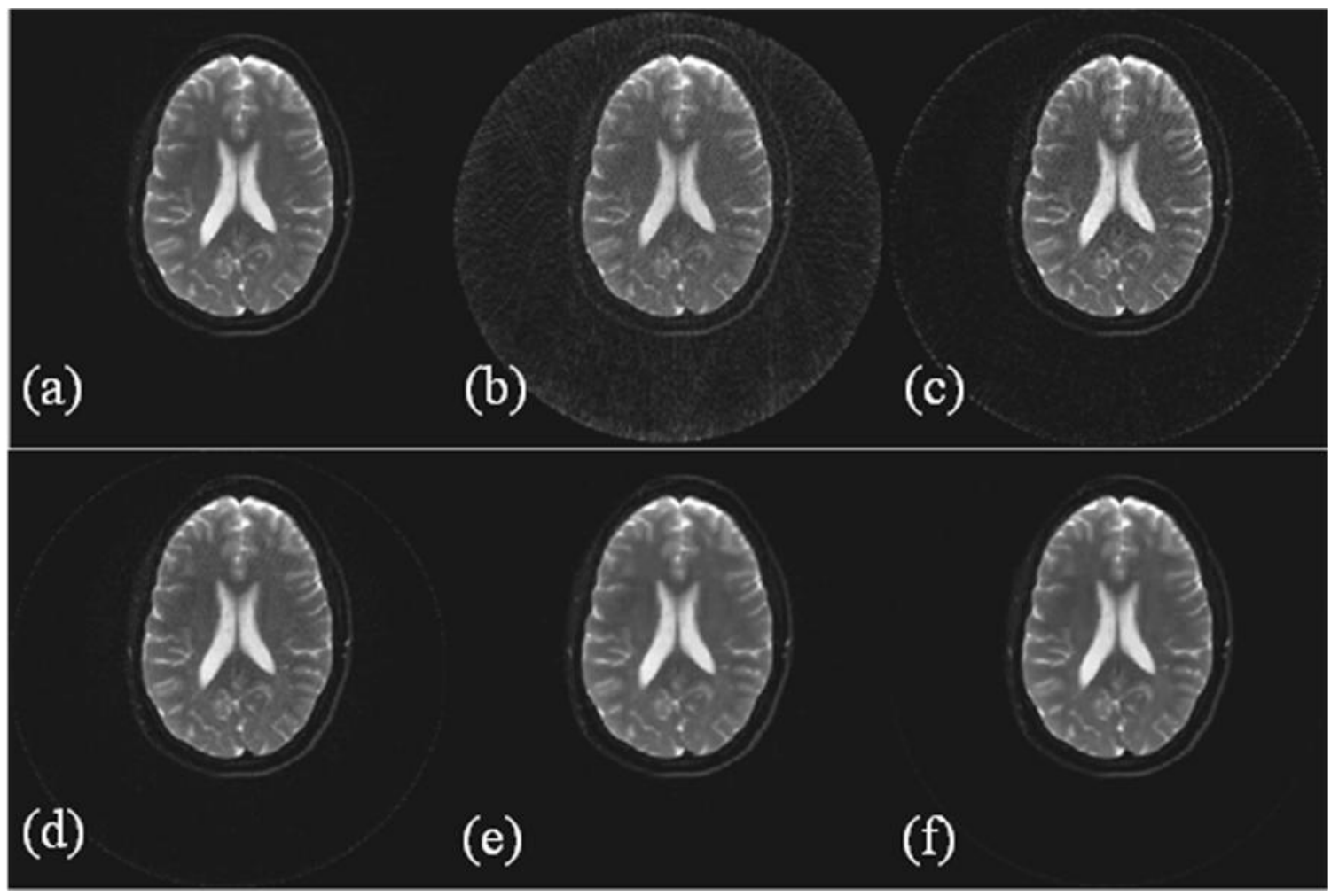

Figure 7: Invivo images reconstructed using different methods. (a) NUFFT+SOS from 256 radial views (b) NUFFT+SOS from 32 radial views (c) CG-SENSE (d) CG-SENSE with Tikhonov regularization (e) CS + SOS (f) PICS. (c)-(e) uses 64 radial views.

\section{CONCLUSIONS}

This paper illustrates that CS and pMRI techniques can be combined. The proposed technique jointly exploits the knowledge of the coil sensitivity profiles together with the sparsity of MRI images. Phantom and in vivo results suggest that such joint processing yields results that are superior to those obtained from independent utilization of CS or pMRI.

\section{REFERENCES}

[1] Sodickson DK, Manning WJ, "Simultaneous Acquisition of Spatial Harmonics (SMASH): Ultra-Fast Imaging with Radiofrequency Coil Arrays," Magnetic Resonance in Medicine, Vol. 38, pp. 591-603, 1997.

[2] Pruessmann KP, Weiger M, Scheidegger MB, Boesiger P, "SENSE: Sensitivity Encoding for Fast MRI," Magnetic Resonance in Medicine, Vol. 42, pp. 952-962, 1999.

[3] Griswold MA, Jakob PM, Heidemann RM, Nittka M, Jellus V, Wang J, Kiefer B, Haase A, "Generalized Autocalibrating Partially Parallel Acquisitions (GRAPPA)," Magnetic Resonance in Medicine, Vol. 47, pp. 120210, 2002.

[4] Blaimer M, Breuer F, Mueller M, Heidemann RM, Griswold MA, Jakob PM, "SMASH, SENSE, PILS, GRAPPA: How to Choose the Optimal Method," Topics in Magnetic Resonance Imaging, Vol. 15, No. 4, pp. 223-36, August 2006.

[5] Larkman DJ, Nunes RG, "Parallel Magnetic Resonance Imaging," Physics in Medicine and Biology, Vol. 52, pp. R15-R55, April 2007.

[6] Candès E, Romberg J, Tao T, "Robust uncertainty principles: exact signal reconstruction from highly incomplete frequency information," IEEE Transactions on Information Theory, Vol. 52, pp. 489-509, February 2006.

[7] Donoho D, "Compressed Sensing," IEEE Transactions on Information Theory, Vol. 52, pp. 1289-1306, April 2006. 
[8] Lustig M, Lee JH, Donoho DL, Pauly JM, "Faster Imaging with Randomly Perturbed Undersampled Spirals and L 1 Reconstruction," Proceedings of ISMRM, 13th Annual Meeting, Miami, Florida, 2005.

[9] Lustig M, Santos JM, Donoho DL, and Pauly JM, "k-t SPARSE: High Frame Rate Dynamic MRI Exploiting Spatio-Temporal Sparsity," Proceedings of ISMRM, 14th Annual Meeting, Seattle, Washington, 2006.

[10] Lustig M, Donoho DL, Pauly JM, "Rapid MR Imaging with Compressed Sensing and Randomly Under-Sampled 3DFT Trajectories," Proceedings of ISMRM, 14th Annual Meeting, Seattle, Washington, 2006.

[11] Chang TC, He L, Fang T, "MR Image Reconstruction from Sparse Radial Samples Using Bregman Iteration," Proceedings of ISMRM, 14th Annual Meeting, Seattle, Washington, 2006.

[12] Bilgin A, Krishnan A, Altbach MI, "Rapid Imaging Using Undersampled Radial Trajectories and L1 Reconstruction" Proceedings of ISMRM, 15th Annual Meeting, Berlin, Germany, 2007.

[13] Song J, Chang TC, Fang T, Speier P, Mueller E, Liu QH, "Practical Iterative MR Image Reconstruction from Very Sparse Radial Samples," Proceedings of ISMRM, 15th Annual Meeting, Berlin, Germany, 2007.

[14] Haldar JP, Hernando D, Sutton BP, Liang ZP, "Image Reconstruction from Randomly Undersampled Cine MRI Data - A Comparison with the k-T BLAST Method," Proceedings of ISMRM, 15th Annual Meeting, Berlin, Germany, 2007.

[15] Lustig M, Donoho D, Pauly JM, "Sparse MRI: The application of compressed sensing for rapid MR imaging," Magnetic Resonance in Medicine, Vol. 58, pp. 1182-1195, 2007.

[16] Block KT, Uecker M, Frahm J, "Undersampled Radial MRI with Multiple Coils. Iterative Image Reconstruction Using a Total Variation Constraint," Magnetic Resonance in Medicine, Vol. 57, pp. 1086-1098, 2007.

[17] Pruessmann KP, Weiger M, Bornert P, Boesiger P, "Advances in Sensitivity Encoding With Arbitrary k-Space Trajectories," Magnetic Resonance in Medicine, Volume 46, Issue 4, pp. 638-651, 2001.

[18] Hestenes MR, Stiefel E, "Methods of Conjugate Gradients for Solving Linear Systems.," Journal of Research of The National Institute of Standards and Technology, Vol. 49, pp. 409-436, 1952.

[19] Lin FH, Kwong KK, Belliveau JW, Wald LL, "Parallel imaging reconstruction using automatic regularization," Magnetic Resonance in Medicine, Vol. 51, pp. 559-567, 2004.

[20] L. Ying, D. Xu, and Z.-P. Liang, "On Tikhonov regularization for image reconstruction in parallel MRI," in Proceedings of the International Conference of the IEEE Engineering in Medicine and Biology Society, pp. 10561059, 2004.

[21] Hoge WS, Kilmer ME, Haker SJ, Brooks DH, Kyriakos WE, "Fast Regularized Reconstruction of Non-Uniformly Subsampled Parallel MRI Data," Proceedings of IEEE International Symposium on Biomedical Imaging (ISBI06), pp. 714-717, 2006.

[22] Hansen PC, Rank-Deficient and Discrete Ill-Posed Problems: Numerical Aspects of Linear Inversion, Philadelphia; SIAM, 1998.

[23] McKenzie CA, Yeh EN, Ohliger MA, Price MD, Sodickson DK, "Self-calibrating Parallel Imaging with Automatic Coil Sensitivity Extraction,” Magnetic Resonance in Medicine, Vol. 47, pp. 529 - 538, 2002.

[24] Candès EJ, Tao T, "Near-Optimal Signal Recovery From Random Projections: Universal Encoding Strategies," IEEE Transactions on Information Theory, Vol. 52, pp. 5406-5425, 2006.

[25] Candès EJ, Romberg J, "Sparsity and Incoherence in Compressive Sampling," Inverse Problems, Vol. 23, pp. 969985, 2007.

[26] Glover GH, Pauly JM, "Projection reconstruction techniques for reduction of motion effects in MRI," Magnetic Resonance Medicine, Vol. 28, pp. 275-289, 1992.

[27] Fessler JA, Sutton BP, "Nonuniform Fast Fourier Transforms using min-max Interpolation," IEEE Transactions on Signal Processing, Vol. 51, No. 2, pp. 560-74, 2003. 